Voix et Images

volxetimages

\title{
Prisonnier dans ce trou, ce Trou de mémoire
}

\section{Anthony Wall}

Volume 14, numéro 2 (41), hiver 1989

L’édition littéraire au Québec

URI : https://id.erudit.org/iderudit/200777ar

DOI : https://doi.org/10.7202/200777ar

Aller au sommaire du numéro

\section{Éditeur(s)}

Université du Québec à Montréal

\section{ISSN}

0318-9201 (imprimé)

1705-933X (numérique)

Découvrir la revue

\section{Citer cet article}

Wall, A. (1989). Prisonnier dans ce trou, ce Trou de mémoire. Voix et Images, 14(2), 301-320. https://doi.org/10.7202/200777ar d'utilisation que vous pouvez consulter en ligne.

https://apropos.erudit.org/fr/usagers/politique-dutilisation/ 


\title{
Prisonnier dans ce trou, ce Trou de mémoire
}

\author{
par Anthony Wall, Université de Calgary
}

Comme cela est étrange, je veux dire: comme ma situation me parait soudain le contraire même d'une situation privilégiée. Je n'ai pas le dessus dans cette affaire; je suis en dessous, loin derrière, dans un état voisin de l'égarement.

Hubert Aquin, Trou de mémoire, 1968.

\section{La dispersion dans la diégèse}

Le trajet que doit suivre une lecture attentive de Trou de mémoire passe sous l'influence d'un grand mouvement actif au niveau de l'espace diégétique. Dans Trou de mémoire, éclate d'une manière spectaculaire tout centre qui pourrait tant soit peu ancrer le mouvement du récit. Ce roman vit d'une multiplication des voix narratives d'un genre beaucoup plus osé que le «je» tridimensionnel qui nous raconte les péripéties de Prochain Épisode, par exemple. Des niveaux diégćtiques s'accumulent, s'intègrent les uns aux autres, se contredisent, se contestent. Si dans Prochain Épisode les récits et les cadres géographiques conduisent souvent le lecteur à se demander où il est, dans Trou de mémoire, le lecteur en vient à perdre tout point de repère avec le monde où il a l'habitude de vivre.

Contrairement à ce qu'avancent certaines analyses récentes ${ }^{1}$, Trou de mémoire est incontestablement lié à la référentialité québécoise qui lui sert de fondement. La nature des liens réunissant le texte et cette référentialité est pourtant très complexe. Il y est toujours question de cette révolution tant rêvée. Et pourtant, cette référentialité est ici textualisée d'une manière déroutante, provocatrice, incontrôlable. Dans Trou de mémoire, le lecteur est même forcé de vivre cet état de déroute, cette provocation, ce tourbillon sans contrôle, et de les vivre de l'intérieur.

Ce roman est avant tout une enquête sur les notions difficiles de vérité et de réalité, telles qu'elles sont vécues par un être qui ne peut plus se satisfaire d'une vie égale, maîtrisée, calculable. Rien n'est plus égal à rien, il n'y a plus de maî-

1 Nous citons, à titre d'exemple, l'étude de René Lapierre, l'Imaginaire captif. Hubert Aquin, Montréal, Quinze, 1981, p. 59, 62 (Prose entière). 
trise possible, tout passe hors des limites du vérifiable. La lecture de Trou de mémoire se trouve constamment bouleversée, car les structures rassurantes qu'elle tente de mettre en place sont à tout moment décimées par d'autres éléments contradictoires du texte. En ceci, l'expérience du lecteur désemparé, sans point de repère, résulte de l'expérience collective à laquelle ressortit la situation énonciatrice. W. Krysinski, par exemple, voit partout dans l'œuvre aquinienne des lieux conflictuels, contradictoires, lieux qui fournissent souvent à ces textes les liens complexes qu'ils entretiennent entre leur contenu symbolique et leur valeur référentielle:

Les romans d'Aquin renvoient à une subjectivisation imaginaire de l'expérience collective et, par là même, ils atteignent au statut d' une forme symbolique qui a partie liée avec une collectivité. L'inscription de cette forme dans le texte conditionne les diverses modalisations de son ouvre qui en font un signe compris comme lieu conflictuel. ${ }^{2}$

\section{La vérité vitale contre la vérité stratifiée}

Repenser la notion de vérité équivaudra ici à la concevoir comme relevant de l'expérience mouvementée de la vie. Appelons-la la vérité vitale, vérité qui doit être vécue, vérité de la vie donc. Cette vérité dans laquelle baignent tous les personnages de Trou de mémoire, et par extension le lecteur, est une condition qui pourrait se comparer, conformément au titre du roman, à un énorme trou d'où il est possible de sortir, trou dans lequel tout le monde est prisonnier. Les personnages essaient les uns après les autres de s'extraire de ce mouvement vital de la vérité, de s'en extraire à tout prix, même au prix d'un suicide. La leçon à tirer de leurs multiples échecs est simple: on vit la vérité, on ne l'observe pas d'une quelconque position objective. En d'autres termes, on ne s'extrait pas de la vérité.

La tragédie que vivent l'éditeur, Olympe Ghezzo-Quénum et Pierre X. Magnant est précisément l'entreprise folle d'essayer de dominer la vie de l'extérieur. La vérité est pour eux une entité finie, cernable, définissable. Il suffirait, croient-ils, de se hisser, telle la fausse RR de «Semi-finale» ${ }^{3}$, dans une position privilégiée d'observation détachée, soit le «théâtre supérieur», soit le rôle d'un' éditeur, soit la position du narco-analyste, soit la place de l'auteur du crime parfait, pour pouvoir dominer la vie, lui dicter sa position souvent secrète, lui imposer de l'extérieur un ordre qui n'est pas le sien. Et voilà que malgré toutes ces tentatives menées pour arrêter le cheminement des sens du roman, on finit toujours par retomber dans le trou, telle la fausse RR retombant sur la scène, car la vérité, elle, n'arrête jamais de se déplacer. Les efforts fantastiques conçus pour l'entraver ne font qu'accélérer son mouvement et donner le vertige.

2 Wladimir Krysinski, Carrefours de signes-essais sur le roman moderne, La Haye, Mouton, 1981, p. 347.

3 Hubert Aquin, Trou de mémoire, Montréal, Cercle du livre de France, 1968, p. 123-134. Toute référence ultérieure à cet ouvrage sera suivie uniquement du numéro de page entre parenthèses. 
Trou de mémoire présente une série de tentatives pour conquérir la vérité de la vie par la tactique de la division. En ceci on pourrait dire qu'il reflète sur le plan théorique la notion du type russellien ${ }^{4}$ et surtout la «conception sémantique» de la vérité telle qu'élaborée par $\mathrm{A}$. Tarski ${ }^{5}$. C'est surtout cette dernière formulation théorique qui retiendra ici notre attention.

Fonctionnant donc dans une ligne de pensée qui a aussi donné naissance au type russellien, la conception sémantique de la vérité a été proposée par Tarski pour faire face à deux problèmes fondamentaux de la sémantique philosophique anglo-saxonne: comment éviter les paradoxes sémantiques; comment donner des bases solides à la sémantique sans tomber dans la pétition de principe. Ici repose toute la difficulté. La vie n'est jamais «résolue» si nous nous limitons à hiérarchiser l'expérience en niveaux de vérité. Pour nous, Tarski exemplifie à merveille le genre de pensée auquel s'en prennent bon nombre de stratégies textuelles de Trou de mémoire. Il voit dans le paradoxe quelque chose à éviter, quelque chose à résoudre, quelque chose à prendre avec les pincettes de l'analyse objective. Tout problème découlant d'un quelconque paradoxe peut être affronté si on a recours à la stratégie de la stratification opérée sur le concept de la vérité. Pour Russell et Tarski, la vérité d'une affirmation donnée ne pourra être décidée que de l'extérieur de son plan d'énonciation, c'est-à-dire en citant avec l'usage d'un métadiscours cette même affirmation. Dire d'une phrase qu'elle est vraie, ce serait pour eux se hisser au-dessus d'elle afin de la juger à partir d'un langage nouveau, objectif par rapport à la phrase d'origine ${ }^{6}$. La «vérité» ne serait donc nullement un concept unitaire. C'est ici plutôt toute une série de lexèmes homonymiques qui se multiplient infiniment chaque fois qu'un locuteur ou un philosophe veut se prononcer sur la véracité d'un énoncé quelconque. Chaque méta-niveau nécessite un discours séparé des autres niveaux qu'il traite et ce n'est que la langage ordinaire, «quotidien», cette chose imparfaite, qui essaie d'assimiler sous une seule notion toutes les stratifications de l'étiquette linguistique en question.

Chez Tarski, ces deux principes sont explicites:

Il faut toujours penser la notion de vérité, et avec elle celle de la phrase, en termes d' une langue particulière. ${ }^{7}$

4 Voir les trois ouvrages fondamentaux qui suivent: Bertrand Russell, An Inquiry in Meaning and Truth, Londres, MacMillan, 1940; Logic and Knowledge, Londres, George Allen and Unwin, 1956; et surtout B. Russell et A. N. Whitehead, Principia Mathematica, Cambridge, Cambridge University Press, 1925.

5 Nous pensons aux articles suivants: Alfred Tarski, «The Semantic Conception of Truth and the Foundations of Semantics», Philosophy and Pnenomenological Research, 4, 1944, p. 341-376; «The Consept of Truth in Formalized Language», Logic, Semantics, Mathematics, New York, Oxford University Press, 1956, p. 152278; «Truth and Proof», Scientific American, 220, 1969, p. 63-77.

6 Voir à ce propos Hartry Field, «Tarski's Theory of Truth», Mark Platts ed. Reference, Truth, and Reality, Londres, Routledge \& Kegan Paul, 1980, p. 83100 , et surtout les pages $90-91$.

7 Alfred Tarski, «The Semantic Conception of Truth and the Foundations of Semantics», loc. cit., p. 342. Notre traduction de la phrase: We must always relate the notion of truth, like that of a sentence, to a specific language. 
Nous nous voyons donc dans l'obligation de comprendre qu'il s'agit alors, non pas d' un concept unitaire, mais de plusieurs concepts qui sont dénotés au moyen d' un seul mot. 8

De ce point de vue, les personnages de Trou de mémoire sont de fervents tarskistes. Ils multiplient les niveaux diégétiques nécessaires pour véhiculer tant de méta-discours. C'est ainsi qu'ils essaient d'échapper aux lois implacables du paradoxe, mais ils découvrent enfin que le paradoxe ne s'enraie pas aussi facilement. Trou de mémoire vit même de paradoxes et de contradictions qui, eux, sont inépuisables. Le paradoxe fait partie intégrante de la vie, de la vérité. Et c'est ce que les personnages du roman n'arrivent pas à accepter. Il y a tout de même une limite à l'imposture; et celle de la présumée RR est criarde et de fort mauvais goût. (p. 136)

Si nous pouvons tirer une autre leçon de Trou de mémoire, ce serait celleci: il n'y a tout simplement pas de limites à assigner à la paradoxale vérité. La stratification de la vérité ne résoud en rien les problèmes relevant du paradoxe, car le paradoxe est par définition inépuisable. De tous les personnages du roman, seule RR, celle qui a perdu la mémoire, échappe involontairement aux meurtres et aux suicides. Elle apprend à vivre à l'intérieur de la vie paradoxale en l'acceptant pleinement, en s'y adaptant même. Car le paradoxe, lui non plus, n'a pas de mémoire. Il se répète infiniment sans avancer. C'est seulement la mémoire qui le rend invivable. Mais si nous oublions, ainsi que le fait $R R$, tout ce qui a déjà été répété, il nous deviendrait presque insupportable.

Nous verrons plus loin, lors de notre approche de l'écriture comme geste permanent, que la victoire de RR consiste en ceci qu'elle réussit à vivre son passé au présent; que son passé n'est plus coupé de son présent comme s'il appartenait à une autre dimension hermétiquement fermée; que son avenir, présent sous la forme d'un fotus, est littéralement agissant sous la forme de «compartimentage» du vécu, que ce soit celui de la vérité ou celui de la temporalité. Le temps n'est plus divisé en trois petites cases étiquetées passé, présent ou futur. Selon nous, une telle catégorisation ne fait que reproduire en termes temporels toutes les stratégies que nous analyserons plus loin à l'aide de la notion de stratification. Le temps se vit de l'intérieur?.

\section{L'objectivité trouée}

Dans Trou de mémoire, la vérité tarskienne est une vérité qui se rapproche sensiblement de celle du roman policier. Elle a pleinement confiance en la

8 Ibid., p. 355. Notre traduction de la phrase: We should reconcile ourselves with the fact that we are confronted not with one concept, but with several different concepts which are denoted by one word.

9 Il est tentant, dans ce contexte, de faire appel à la question de Jean-François Lyotard qui évoque la situation pénible de l'Allemagne moderne face à son histoire noire de l'époque nazie: Peut-il y avoir progrès sans anamnèse? L'anamnèse conduit, à travers une douloureuse élaboration, à élaborer le deuil des attachements, des affections, de toutes sortes, amours et ferveurs, qui se sont associés à ces noms. JeanFrançois Lyotard, le Postmoderne expliqué aux enfants, Paris, Galilée, 1986, p. 131. 
possibilité de l'objectivité. Un problème se dessine: ce que l'on appelle l'objectivité n'est qu'un méta-discours qui, lui, n'est pas moins sujet à la hiérarchisation des niveaux de vérité que les phénomènes sur lesquels il se prononce. Mais ici encore, le terme «hiérarchisation» est quelque peu mal placé, car nul niveau métadiscursif, nul langage, nul niveau diégétique n'est mieux qualifié que d'autres pour atteindre la vérité objective. Car la vérité ne s'obtient pas, elle se vit.

D'où tous ces problèmes des personnages et du lecteur désemparés de Trou de mémoire. La confiance totale en la possibilité de l'objectivité est nuisible à celui qui partage cette confiance, car que se passerait-il si cette confiance s'avérait mal fondée? C'est justement la position du lecteur face à l'éditeur du livre. L'éditeur est finalement un personnage sujet à l'erreur comme tous les autres personnages. Le lecteur s'appuyant sur les connaissances objectives de l'éditeur sera vite dérouté:

Comment le lecteur pourra-t-il se reconnaître si je perds pied, moi l'éditeur, dans ce courant désordonné de chapitres et d'interprétations? (p. 137)

Me voilà, moi éditeur, aux prises avec des mots, des phrases et des pensées qui n'ont de valeur que si je réussis à les formuler dans un corpus logique et selon un ordonnement consistant. C'est l'ironie du sort! (p. 139)

Son ordre ne survit même pas jusqu'à la publication, car il semblerait que l'ordre final soit plutôt celui de RR ([...] selon l'ordre que je lui ai inculqué et dans la sucession que j' ai choisie [...], p. 201). Et pourtant, il y a toujours des notes non signées (p. 121, 126, 127, 129, 132, 193). S'agirait-il alors d'un troisième, d'un quatrième ou même d'un cinquième éditeur? La question est ouverte!

En effet, tous les personnages sont sujets à l'erreur. Nous discernerons assez facilement des erreurs, des contradictions, des inexactitudes dans les discours de chaque personnage. L'éditeur, par exemple, se complaît souvent à signaler des «erreurs» ou, du moins, les inexactitudes contenues dans le récit de Pierre X. Magnant. Lui-même n'est pourtant pas moins victime de quelques inexactitudes quand il parle, par exemple, des nombreuses études de Fribourg-Blanc sur la simulation (p. 105), car Fribourg-Blanc a surtout écrit à propos de problèmes de psychologie chez les soldats. D'où, d'ailleurs, la piquante ironie à l'endroit du terme de la simulation, RR signale d'autres inexactitudes dans le discours et dans les notes mêmes de l'éditeur. Mais son discours à elle n'est pas non plus sans tache. Elle nous laisse comprendre, par exemple, que le Blason de la mort et les Ambassadeurs se trouvent côte à côte à la National Gallery à Londres, ce qui n'est pas le cas (p. 132). Ensuite, viennent au bas de la même page deux notes pleines d'inexatitudes, notes non signées d'ailleurs, qui soulèvent encore plus de problèmes ${ }^{10}$. Le discours d'Olympe Ghezzo-Quénun contient également de

10 S'agit-il d'une erreur de la part de RR, qui prend un masque dans ce chapitre de la «Semi-finale», ou de la part d'un autre qui nous reste inconnu? En tout cas, remarquons que le tableau est catalogué sous le numéro 1314 , non pas 314 , que le 
nombreuses maladresses. A lire, par exemple, la page 151, on croirait que le Niger se jette dans la mer à Lagos, ce qui est faux. Ou quand il parle, à la page 154, du décalage horaire entre la Suisse et le Nigéria, il oublie qu'il n'y en a aucun. De telles inexactitudes existent dans tous les discours, dans la narration et dans les notes. Mais la chose la plus fascinante dans ce dossier est la façon dont nous, lecteurs du texte, sommes en mesure de découvrir ces inexactitudes. Souvent, des erreurs nous sont tout simplement signalées dans le texte par des notes du genre: Ce terme doit sans doute se référer aux hypothèses émises sur l'ecmnésie; et à ce titre, il est impropre. (p. 177) Il s'agit souvent de détails techniques que très peu de lecteurs connaîtront. Nous sommes donc souvent tentés par paresse d'accepter de telles déclarations, mais ce sont des modalisateurs comme «sans doute» qui font problème. Ici, le mot qui a suscité la note, «antérograde», est peut-être mal utilisé comme l'indique la note en question. On parle plutôt d'amnésie antérograde. Mais s'agit-il ici effectivement d'ecmnésie11? Revoyons le texte qui donne naissance à cette note:
Ah, j'aurais mieux fait de ne pas lui poser de questions. Car elle a recommencé comme l' autre jour quand nous étions dans le Cisalpin; et ces récapitulations infinitésimales mais toujours inachevées me rendent malades. Je suis vidé, complètement vidé? RR est couchée sur le côté, tandis que moi je veille, obsédé par le dénouement qui manque toujours à son récit antérograde (1), obsédé par ce cauchemar sériel qui m' a ravi celle que j'aime. (p.177)

Ce sont précisément les modalisateurs du genre «sans doute» qui devraient nous mettre la puce à l'oreille. Quand, dans une note signée du nom de RR, nous lisons: Et quiconque veut vérifier mon assertion n'a qu'à ouvrir à la page 185 , l'ouvrage de Blanchot, intitulé Études critiques (Gonthier, Paris-Genève, 1961) (p. 78), il faut bien sûr relever le défi pour découvrir, du fait que ce livre n'existe pas, que Blanchot n'a pas commis cette phrase. C'est plutôt Roland Barthes qui l'aurait écrite dans une lettre personnelle envoyée à Aquin ${ }^{12}$.

\section{La tyrannie de l'authentification}

Un régime de doute s'instaure tout lentement dans la lecture de Trou de mémoire. Et le doute, comme la fiction, est contagieux. Quand RR me donne une définition du mot «hypomimique» à la page 170 , je doute car je ne la trouve nulle part attestée: Quand elle me dit, à la même page, que l'expression latine tådium

rapprochement entre le Blason et les Ambassadeurs a été signalé par R.N. Wornum, non pas W. Wornum et que c'est P. Ganz, non pas W.W. Gans, qui a écrit Hans Holbein der Jüngere, livre publié à Bâle en 1949, non pas à Breslau en 1913. Nous verrons plus loin le problème des notes correctives qui sont, elles aussi, fausses.

11 Ecmnésie: $n$.f. (gr. ek, hors de, et -mnésie) Psychiatr. État au cours duquel le sujet revit des scènes entières de son passé comme si elles étaient présentes. (L'ecmnésie se rencontre dans l'épilepsie, l'hystérie et parfois dans l'onirisme.) Grand Dictionnaire encyclopédique Larousse, tome IV, Paris, Larousse, 1983, p. 3528.

12 Fait signalé par Jean-Pierre Martel, «Trou de mémoire: cuvre baroque (essai sur le dédoublement et le décor)», Voix et images du pays, VIII, 1974, p. 75. 
vitce vient du poète Horace, je doute et je découvre plutôt qu'elle est attestée chez le grammairien Aulus Gellius (mort en 180 de notre ère) dans ses Noctes Atticæ (vii.xviii) ${ }^{13}$, chez P. Papinius Statius (45-96 de notre ère) dans son Thebias (7.464) et chez L. Annæus Seneca (né en 55 av. J.-C.) dans ses Suasoriæ (7.4)14. C'est que le système de notes du texte est extrêmement arbitraire: quelques notes sur certains québécismes (p. 70), mais d'autres ne sont jamais indexés. Quelques notes sur certains termes obscurs, alors que d'autres sont laissés de côté. Et pourquoi nous expliquer le sens affectif du mot «patriote» (p. 89), quand tant d'autres mots passent sans note?

Nous nous rendons compte que les notes servent moins à «authentifier» (pour reprendre un terme cher à l'éditeur) le texte qu'à contester l'entreprise même d'authentification du récit. Car celle-ci, ou du moins la tentative d'y parvenir, présuppose un point de vue objectif, privilégié, supérieur par rapport à la chose à vérifier. C'est une entreprise essentiellement tarskienne. Chose intéressante ici, le point de vue le plus souvent privilégié, celui de RR, est justement le plus erratique. Or, il faut signaler à propos de mes propres tentatives de vérification des multiples notes et des faits peu connus cités dans Trou de mémoire que le doute est généré à l'intérieur du texte, à partir de quelques «fausses notes», mais que le «faux» ne peut être constitué comme tel qu'à partir de sources extra-textuelles. En dépit des critiques d'Olympe Ghezzo-Quénum, le doute systématique foncièrement cartésien fonctionnera pour ce texte comme une machine extrêmement efficáce destinée à incorporer au texte des sources venant de domaines hétéroclites. Il s'agit enfin d'une entreprise intimement lice au problème de l'illusion référentielle: c'est uniquement grâce à l'ancrage bien solide de Trou de mémoire dans une situation localisable que la construction du «faux» devient concevable. Mais elle devient dès lors tout à fait problématique: car si ce roman pousse toujours plus loin les limites de sa propre textualité, comment serait-il possible de constater le faux à partir du hors-texte quand ce dernier est constamment incorporé au texte?

Les personnages de Trou de mémoire cherchent souvent, et parfois même désespérément, à tout authentifier. Même le manque d'authentification peut devenir ici source d'authentification. Tel Monsieur Teste de Paul Valéry, Pierre $\mathrm{X}$. Magnant n'est archi-connu que dans la mesure où il est resté inconnu:

Les activités terroristes de P.X. Magnant font maintenant partie de l' histoire secrète du terrorisme québécois. S'il n'a pas fait les manchettes, cela est finalement tout à son honneur; c'est qu'il n'a jamais été même soupçonné. (p. 118)

13 Tel qu'attesté dans Charlton Lewis et Charles Short, A Latin Dictionary, Oxford, Clarendon Press, 1966, p. 1 384. Remarquons aussi que la même attribution fautive à Horace se trouve dans Hubert Aquin, l'Antiphonaire, Montréal, Cercle du livre de France, 1969, p. 58.

14 P.G.W. Glare, Oxford Latin Dictionary, Oxford, Clarendon Press, 1982, p. 1900. L'expression tadium vitae n'est nulle part attestée dans le Lexicon Horatianum de Dominicus Bo, Hildesheim, Georg Olms Verlagsbuchhandlung, 1966, 2 vol. 
C'est que cette machine à tout incorporer dans le but de s'authentifier en arrive même à utiliser la fictivité comme gage de l'authenticité. Trou de mémoire est un fascinant jeu de miroirs qui se reflètent les uns les autres, non pas pour cacher l'inanité du roman, mais plutôt pour effacer, en les incorporant à l'intérieur de sa propre textualité, les confins séparant la réalité de la fiction. Mais seule RR comprend à fond ce mécanisme. Les autres personnages se contentent de chercher la vérité ailleurs, soit en essayant de se transporter eux-mêmes vers un ailleurs, soit en se fiant au témoignage d'un autre. En effet, la question d' «un autre» dans Trou de mémoire est un motif qui hante bien des pages du livre. Par exemple:

Un ami qui a des connaissances en paléontologie $m$ 'a fait remarquer que, selon les auteurs modernes, les singes $R$ hésus ne sont pas dans la catégorie des Primates. Cet ami, à qui j'ai fait lire les passages où Pierre X. Magnant décrit les singes du laboratoire Redfern, de l'université McGill, croit plutôt que les singes «voyeurs», mentionnés dans le manuscrit, sont vraisemblablement des Gibbons (appelés aussi Wou-Wou) originaires de Java ou de Bornéo, ou bien des Chimpanzéa Tchégo dont l'espèce prolifère en Basse-Guinée et jusqu' en Oubangui. (p. 27)

Cette démarche est typique dans le roman: chercher toujours dans l'autre ou l'ailleurs l'authentification ou la vérité. Chercher non pas à vivre la vérité, mais à s'en séparer, manœuvre révélatrice de la perspective théorique selon laquelle la vérité est une entité stratifiée. Notons en outre dans ce dernier détail que le recours à l'autre est doublement effectué: l'ami cité en régime indirect a recours, lui, aux «auteurs modernes». Un autre exemple: Je me suis permis de solliciter une expertise psychiatrique d' un médecin-psychiatre dont j'ai raison de croire qu'il est objectif par rapport au récit de Pierre X. Magnant. (p. 20). Remarquons que cette entreprise de vérification chez l'éditeur souffre d'un défaut important. Celui-ci ne révèle jamais l'identité de cet ami. Comment alors vérifier nous-mêmes ce témoignage? Un privilège est ainsi ôté au lecteur qui voit s'appliquer des freins au mouvement de ses propres recherches. Taire la source a ici pour effet secret d'imposer arbitrairement une limite à la vérification et peut par conséquent être perçu comme une nouvelle atteinte à la vérité en tant qu'expérience. C'est d'ailleurs le même problème pour le cas suivant:

Mais un ami confesseur m'a soudain ouvert les yeux; du moins, il m'a fourni de précieux conseils... Je fais grâce au lecteur de mes tâtonnements et j'en arrive à l'essentiel: j'ai finalement utilisé une grille anamorphique pour relire le manuscrit, et voici ce que m'a révélé cette expertise catoptique. (p. 141)

La «grâce» dont il est ici question, aussi bien que la décision prise en coulisse de taire le nom de tant d'amis experts, ont pour effet final de contrarier à tout jamais notre manie cartésienne de vouloir à tout prix vérifier. Mais vérifier toujours de l'extérieur. Et même à une occasion, lorsque le nom du témoin Luigi, maître d'hôtel du restaurant Neptune, est en effet fourni dans le texte, nous ne sommes finalement pas plus avancés. L'éditeur n'en souligne pas moins à maintes reprises l'autorité de Luigi dans cet incident au Neptune: et je prends 
sa parole (p. 76); selon le dire de Luigi; Luigi m'a affirmé que; Luigi a pris un client à témoin (p. 77); En fait, il s'agit dans ce passage de l'importance, sur le plan de la signification du récit, que revêt cette ellipse curieuse qu'on essaie de remplir. Mais chacun vit l'expérience en voyeur, par procuration donc, phénomène typique de l'attitude qui veut stratifier la vêrité de la vie. Et cette expérience menée par procuration atteint ici à des niveaux de complexité assez inusités: témoignage de Luigi qui prend à témoin un client, témoignage de l'éditeur qui commente la version trouée de P.X. Magnant, notre propre lecture. Est-ce là une structure propice à l'idéal empirique du vérifiable?

Voilà justement la faille de cet idéal de la vérité selon lequel on pourrait tout vérifier de l'extérieur. Car une fois commencée, la stratification peut logiquement s'arrêter à aucun endroit en particulier. Quelque chose, quelque part, viendra toujours miner la soi-disant objectivité de la position méta-discursive qu'on adoptera. Ce quelque chose, souvent innommé, se promène partout dans les pages de Trou de mémoire. Toute présence est constamment ruinée par une autre présence qui la double:

Le «double passé» des pays africains est une notion-clé de leur réalité politique. Cette allusion rapide et non appuyée, faite par P.X. Magnant, sonne un peu faux dans le contexte. Je suis tout près de croire que cette phrase savante a été rajoutée par un autre que P.X. Magnant. (p. 88)

Elle me suppliait de l'embrasser longtemps, et encore; elle voulait sentir sa langue (celle de l'autre) lui lécher la peau du ventre, et ses mains (à lui - et non les miennes!) lui chatouiller tendrement la naissance des seins et puis encore ailleurs, les cuisses, là tout en haut, près de la vulve. J'étais étendu sur le lit juste à ses côtés; et j'entendais ses plaintes (adressées à un autre que moi...). (p. 182)

Pourquoi donc persister avec tant d'acharnement à tout authentifier là où tout est déjà dédoublé? À deux reprises sont évoqués des doubles suicides dans le roman, celui de Stefan Zweig et de sa compagne (p. 118-119) ${ }^{15}$ et, plus loin, celui de Heinrich von Kleist et de sa fiancée (p. 151). Le double est partout dans le texte, et avec le champ sémantique de la notion de vérité, il constitue un des réseaux de métaphores transphrastiques les plus importants du roman. On parle, par exemple, des effets secondaires des drogues, du bilinguisme (p. 28), de la deuxième ville française du monde (p. 35, 193), d'un lit doublement double (p. 47), de nos deux corps, d'un pays ambigu, de Joan, à deux doigts de la mort (p. 87), du double passé, des revenants, des masques de morts qui se dédoublent à l'infini dans le ventre des miroirs (p. 128), du double portrait (p. 133), de la seconde phase du livre (p. 144), du récit double, de deux jours (p. 151, 169, 171, 172), de deux heures (p. 153), de deux valises Samsonite (p. 156), de cette nuit vers deux heures (p: 166), et j'en passe. Tout ce qui a l'air simple, unitaire, est au fond déjà

15 Stefan Zweig dont la patronyme contient déjà, de par son étymologie, le «deux» implicite dans l'idée de l'autre, c'est à dire le «zwei» de la langue allemande. 
dédoublé. Quand l'éditeur questionne, par exemple les connaissances prétendument profondes en géographie de P.X. Magnant sur la zone littorale de l'Afrique occidentale, il faut ensuite questionner les sources de l'éditeur. Se limitent-elles au manuel scolaire de Perkins et Stembridge ou se peut-il que ce soit l'éditeur, lui-même, qui ait poursuivi RR dans ces pays, d'où ces connaissances détaillées? Le problème de la vérité qui se fonde sur l'authentification d'une autre n'est nulle part plus manifeste que dans l'exemple de la discussion du portrait des deux Ambassadeurs. Car RR se fonde bien évidemment sur la première édition du livre de J. Baltrusaitis ${ }^{16}$ quand elle écrit:
Alors commence le deuxième acte: le visiteur, repu des apparences infinitésimales du portrait mystérieux, se retire par la porte de gauche, la seule issue de cet enclos. Alors, juste avant de franchir le seuil et de quitter cette chambre obscure, il jette un dernier regard, furtif, sur le tableau et comprend d' un seul coup son double sens: le discernement visuel estompe la figure des «Ambassadeurs» et révèle la «figure cachée». (p. 133)

Mais RR se trompe, comme l'avait fait en premier Baltrusaitis, source qu'elle ne cite pas. Dans la deuxième édition du livre de Baltrušaitis ${ }^{17}$, ce dernier écrit dans le passage correspondant non plus «gauche» mais «droite», erreur qu'il avoue, d'une façon très appropriée pour notre étude de Trou de mémoire, dans une note (la note 32 du chapitre 7). Trou de mémoire a été composé avant la parution de la deuxième édition, mais le fonctionnement du système d'authentification par strates successives ne semble pas moins faire entrer cette deuxième édition dans sa propre textualité fondée sur l'affrontement de différents niveaux diégétiques, de différents niveaux de vérité. C'est ici un exemple merveilleux de la façon dont ce roman incorpore tout, intratextuel et extratextuel, dans sa propre vie textuelle. Ainsi voyons-nous dans quelle mesure il est juste de dire que dans Trou de mémoire, «vérifier» commence à vouloir dire «vivre l'expérience».

Le problème du deuxième degré est déroutant, mais il est aussi d'une importance capitale dans le fonctionnement de la textualité de Trou de mémoire. Ce problème est finalement lié à celui de la notion même de trou. Trois fois, par exemple, il y a des notes dédoublées: elles sont à chaque fois signées de la main du personnage aux initiales doubles, $R R$, la double révolutionnaire. Ces notes se trouvent aux pages 49,140 et 150 du roman. Dans les notes doubles des pages 140 et 150, RR ne cite aucune source et, compte tenu du doute qui s'est installé dans notre lecture, on a du mal à croire ce qu'elle dit à la page 140 à propos de Michel-Ange qui professait le plus grand mépris pour les présumées méthodes de Dürer, car celui-là utilisait lui-même les principes de la perspective ralentie pour ses fresques de la Chapelle Sixtine ${ }^{18}$. Et on apprend par ailleurs, dans le

16 Jurgis Baltrušaitis, Anamorphoses ou perspectives curieuses, Paris, Olivier Perrin, 1955.

17 Jurgis Baltrǔaitis, Anamorphoses ou magie artificielle des effets merveilleux, Paris, Olivier Perrin, 1969.

18 Ibid., premier chapitre. 
Grand Larousse encyclopédique, que la Divina Proportione de Pacioli date non pas de 1509 mais de 1496. Quant à la nature carrément contradictoire des affirmations diverses de la page 150, RR aurait peut-être bien fait de fournir quelques sources à son appui. Déjà son interprétation de la date 1625 , traditionnellement donnée comme celle de la fondation du royaume des Fon, semble quelque peu curieuse. En tout cas, la note dédoublée la plus étonnante est celle de la page 49 , déjà discutée en partie dans l'article astucieux de S. Söderlind ${ }^{19}$. Mais ce que cette dernière ne signale pas dans son étude, ce sont les erreurs qui contaminent la note prétendument correctrice de RR. En parlant des statues du pont Saint-Ange à Rome, RR nous dit que ce sont, en quelque sorte, des cuvres du Bernin «au deuxième degré» [car] les dix statues colossales d' anges qui ornent ce pont ont été exécutées en 1688 d'après les dessins du Bernin. Or, l'une des statues est carrément une copie d'une statue effectivement sculptée par le Bernin pour le pont, mais retirée par Clément IX pour la protéger. Ces détails, peut-être minutieux et d'apparence superflue quand ils sont examinés un à un, ne sont pas, contrairement à ce qu'en dit Söderlind, sans aucune importance ${ }^{20}$, car ils montrent, vus d'une perspective macrotextuelle, tout le mouvement des contradictions produit par la hiérarchisation des niveaux diégétiques. C'est précisément dans la mesure où les corrections de RR contiennent elles-mêmes des demi-vérités et même des faussetés que tout le dynamisme des strates contradictoires se voit en plein mouvement. Car, il faut le signaler également à propos de cette même note, le pont en question n'a pas été construit en l'an 36, mais en l'an 134 et l'autre statue du Bernin dont il s'agit ici n'est pas appelée la Transverbération de Ste-Thérèse, mais plutôt Sainte Thérèse en extase. Toujours est-il que le principe fondamental de ce mouvement des contradictions d'un niveau dićgétique vers un autre appelle de par sa structure même un recours au hors-texte, qu'il ne fonctionne à pleine capacité que parce qu'une lecture donnée, la mienne ou une autre, participe à ce mouvement en y ajoutant un niveau supplémentaire de vérité qui sera ni plus ni moins digéré par la machine Trou de mémoire. Participer à l'essence de Trou de mémoire, vivre son mouvement sémantique, c'est vivre de près les multiples contradictions en y ajoutant les siennes.

\section{Conquérir le trou}

D'où, encore une fois, l'importance du vocable «trou» dans le titre de ce roman. Aquin a créé avec ce livre une machine puissante qui digère sans dćlai toute nourriture extratextuelle qu'une lecture ose lui fournir. C'est peut-être de cette manière qu'il faut vivre à l'intérieur de ce trou dont les limites s'étendent continuellement. Qui dit trou pense normalement ce trou de l'extérieur, dans la partie pleine du non-trou. Il pense déjà lcs limites de ce trou en essayant de le contourner, de l'éviter, de ne pas tomber dedans, dans le vide, dans le paradoxe, dans le non-sens. Mais si la vérité est effectivement à vivre et non pas à éviter

19 Sylvia Söderlind, «Hubert Aquin et le mystère de l'anamorphose», Voix \& images, vol. IX, no 3, printemps 1984, p. 103-111.

20 Ibid., p. 107. 
dans l'observation détachée, si la vérité est effectivement dans le trou qu'on a auparavant essayé d'éviter, alors tout ce qui était auparavant hors du trou commencera inévitablement à y tomber, et il n'y aura plus moyen d'en sortir, car les limites s'étendront continuellement au fur et à mesure qu'on pensera les franchir.

Et cette éternelle expansion des frontières du trou participe de la nature du paradoxe sémantique que toute cette théorie stratifiante de la vérité essaie religieusement de surmonter. Le texte est produit par une multitude de narrateurs qui s'acharnent sans cesse à remplir tout trou, toute omission, toute ellipse. On semble avoir horreur du vide et on impose de l'extérieur n'importe quoi pourvu que le vide semble se cacher, se remplir - vouloir - fût-çe le néant ${ }^{21}$ :

Cette obsession du viol peut se comprendre, peut-être, dans un contexte de phantasme d' impuissance. Les aveux de P.X. Magnant seraient dès lors, bien fragmentaires. Les omissions supposées finiraient par compter plus encore que ce qui est décrit, faisant de ces confessions le masque d' une confession qui n' est pas faite. (p. 117)

Cette attaque contre le trou, le vide ou le non-sens est menée, d'après les principes de la théorie stratifiante de la vérité, à partir de la devise «diviser pour régner». Sur le plan du texte littéraire, cette politique se traduit par la construction stratifiée de multiples niveaux diégétiques organisés hiérarchiquement. Chaque strate est représentée par un narrateur qui est censé avoir une perspective objective sur le texte qu'il commente. L'éditeur travaille donc les textes de P.X. Magnant et d'Olympe Ghezzo-Quénum, RR travaille le texte de l'éditeur. Et les différents éditeurs semblent en outre posséder différents domaines de spécialisation. Ainsi, le texte déploie au niveau superficiel deux stratégies fondamentales pour compartimenter le monde du trou: une stratégie que l'on pourrait dire horizontale, qui a pour but de diviser le désordre perçu en autant de domaines du savoir (la pharmacie, la parasitologie, la sociologie des colonisés, la psychiatrie, la criminologie, la tauromachie, la géographie, l'histoire de l'art, les lois de la perspective, etc.); et deuxièmement une stratégie verticale qui organise le désordre perçu en plusieurs cercles concentriques gouvernés par les lois de la hiérarchisation des niveaux diégétiques du récit ${ }^{22}$.

Ce compartimentage idéaliste devient très vite la proie de certaines tendances métaphoriques plus profondes du texte. Les réseaux métaphoriques de Trou de mémoire estompent la division hiérarchique des niveaux diégétiques en faisant disparaître les identités stables des personnages (et en faisant que RR et l'éditeur se contredisent l'un l'autre). Ces réseaux affectent également la division horizontale des domaines de savoir qui en viennent à se fondre sous l'influence de diverses stratégies transphrastiques de la métaphoe et tout particulièrement de celle du calembour.

La critique aquinienne a déjà amplement travaillé l'importance des personnages qui sont les doubles les uns des autres. Ainsi, dans Trou de mémoire,

21 Sarah Kofman, Nietzsche et la métaphore, Paris, Payot, 1972, p. 111.

22 Jap Lintvelt, «Modèle discursif du récit encadré», Poétique, no 35, 1978, p. 352-266. 
toute hiérarchisation diégétique devient-elle irrémédiablement pleine de fuites qui rendent impossible une quelconque division hermétique. $R R$, en tant qu'éditrice finale (?), est aussi un personnage dans le récit de Ghezzo-Quénum et elle est dédoublée par sa sœur Joan dans le texte de P.X. Magnant. Les lapsus calami de Ghezzo-Quénum soulignent clairement cette fusion des deux sœurs. L'éditeur travaillant le texte de P.X. Magnant se trouve lui-même travaillé par ce texte et on se demande avec Ghezzo-Quénum s'ils ne sont pas la même personne. Ghezzo-Quénum se dit déjà frère de Pierre $X$. Magnant et il se trouve, par l'effet de la drogue et de la fatigue, dans la peau de son frère-ennemi quand il essaie de faire raconter par RR l'épisode oublié de son viol.

Ce sont des réseaux transphrastiques de la métaphore qui structurent une très grande partie du texte et qui contrarient toute tentative des personnages de hiérarchiser et de compartimenter la vérité vitale. Ainsi seront inextricablement mêlés tous les domaines de savoir érigés par les différents narrateurs. Regardons d'abord quelques exemples simples de métaphores structurales fonctionnant en tant que calembours. Ils se rapprochent du type de réseau que l'on peut facilement observer dans le roman à propos de l'image du cercle (révolution, trou, tour, crâne, hémisphères, etc.) qui fait surface dans tout le roman. Nous étudierons ici d'autres exemples moins évidents. foehn:

Olympe Ghezzo-Quénum arrive en Suisse et se trouve vite touché par le

Oui, je me demande bien ce que nous faisons ici, dans cette haute vallée où souffle le foehn - vent dépresseur qui m'épuise et me tue comme la brise chaude qui souffle sur Grand Bassam sans répit, courbant les arbres noirs qui bordent le littoral de la côte des Esclaves. (p. 148)

Qu'Olympe, dont l'origine ethnique est le peuple fon, soit ainsi atteint par le foehn, dans ce roman qui nous incite à survaloriser tout ce qui est occulte et à édifier interminablement des systèmes de correspondances entre les événements ou entre les hommes - systèmes rigoureusement et absolument invérifiables qui finissent par tout expliquer (p. 8), n'a rien d'étonnant sur le plan du signifiant. Dans ce jeu de mots transphrastique sont nettement soulignés les liens entre l'Afrique et la Suisse, entre le pays colonisé et le pays capitaliste par excellence. Les quelques paragraphes du roman expliquant les relations tumultueuses entre les Fon et Yourouba font croire qué la séquence phonétique $[f o n] /[f \varnothing n]$ n'est pas sans importance dans le roman. D'où un deuxième ancrage dans la thématique de la colonisation effectué à partir de ce même complexe sonore:

Alors, ils restent là, debout, avec leurs apocopes à la main, hébétés, plantés comme des cocus dans une intrigue muette qui, fertile en sousentendus, n'est finalement entendue de personne! On a beau ramper sur les tréteaux; croyez-moi, ce n'est pas une sinécure que de donner la réplique à des aphones et de trouver le ton juste quand tout est silence, même le reste... Le Québec, c'est cette poignée de comédiens 
bègues et amnésiques qui se regardent et s'interrogent du regard et qui semblent hantés par la platitude comme Hamlet par le spectre. (p. 56. Nous soulignons)

Autour du [fon] s'agglutinent alors les problèmes du mutisme et du silence qui, eux, sont aussi à relier à la problématique du trou, car le silence est une espèce de trou dans le bruit: le silence ne peut être conçu autrement que comme un intervalle entre deux cris. (p. 57) Pierre X. Magnant veut enseigner le cri et le juron qui rompent le mutisme passif qui ne mène nulle part. Il préconise donc le bruit, l'équivalent sonore du désordre total, du trou plein par opposition au trou vide du silence, et ceci pour dépasser l'ordre que le silence impose au bruit. La verbalance fonale (p. 15) est donc d'une extrême importance dans le contexte d'un roman qui prêche le bruit et dans cette lignée de pensée, il y a lieu d'évoquer le passage sur le

fameux poisson résurrectionnel qu'on s'empresse de combattre, aussitôt qu'il est inculqué, par une injection surdosée de sulfonal contre-résurrectionnel qui est suivi de près, selon les ordonnances du conquérant, par l'absorption d' un produit antagoniste, et ainsi de suite. (p. 38)

Le [fon] récupère dans son réseau toute la thématique de la pharmacie dans le roman: il est donc doublement lié à la Suisse, lieu connu pour ses produits pharmaceutiques, à la révolution québécoise et aux peuples africains colonisés.

Il serait, bien sûr, facile de questionner la légitimité d'une telle extrapolation faite à partir de quatre mots isolés dans le texte. Si nous nous sommes permis d'inclure une telle discussion qui semble à première vue reposer sur des bases quelque peu labiles, c'est que le cas du [fon] n'est qu'un seul exemple parmi une myriade de procédés qui se combinent dans le texte de Trou de mémoire pour défaire à un niveau profond les compartiments et les hiérarchisations que les personnages s'ingénient à ériger à la surface du texte dans le but de conquérir les trous. Quand, à la page 73, l'éditeur parle dans les termes suivants: Par loyauté pour l'auteur et par respect pour son lecteur, je ne veux pas arranger le récit de Pierre X. Magnant, comme si le lecteur du texte de P.X. Magnant n'était pas en même temps le sien, nous y verrons une de ces multiples tentatives de diviser le monde. Si l'exemple du [fon] n'était pas assez convaincant, nous proposerions un autre exemple de réseau métaphorique qui fournit un nombre étonnant d'occurrences. Il s'établit aurour du lexème /victoire/: il s'agit ici d'un groupement qui traverse plusieurs niveaux et compartiments du texte. Nous y verrons se conjuguer la thématique de la révolution, celle de la défaite, celle du crime parfait, ainsi que d'autres domaines touchés par le texte: la vérité, la violence et le viol. Quelques exemples suffiront ici.

Aux pages 62 et 63 du roman, P.X. Magnant décrit la scène de l'orgasme de Joan devant Buckingham Palace. Il est ici question d'un réverbère victorien qui faisait de nous un couple de malfaiteurs (p. 62. Nous soulignons). Plus loin, nous lisons: $j$ 'ai déboutonné l'imperméable de Joan, afin de trouver l'agrafe latérale de sa robe et, ma foi, j'ai été victorieux partout à la fois. (p. 62. Nous 
soulignons) À la page suivante, il s'agit du règne de Victoria (couronnée en 1837, l'hostie!) (p. 63) Le réseau «victoire» est bien développé par ailleurs dans le texte:

J'ai trouvé dans Mao Tsé-Toung, dans «La guerre révolutionnaire», p. 94: "Il est indispensable de gagner la première bataille». Cette première victoire à laquelle se réfère $P$. X. Magnant, c'est peut-être justement celle des Patriotes à Saint-Denis - et non pas le viol de Joan dont il est question plus haut. Notes de RR. (p. 43)23

Selon les grands historiens de l'art, le chef d' ouvre du Bernin est la statue appelée "La transverbération de Ste-Thérèse». Le fameux groupe sculpté se trouve dans l'église Ste-Marie-de-la-Victoire à Rome. (p. 49)

Holmes a inventé, entre autres choses, la pharmacie posthume: il hantait les laboratoires de Charing Cross et battait les macchabées à coups de canne. Voilà mon homme. Voilà l'ancêtre victorien dont je voudrais tirer ma généalogie coloniale et mon héritage morbide. (i. 81-82)

Et il ne faudrait surtout pas oublier la présence souterraine du théoricien victorien John Ruskin dans ce roman complexe. C'est en effet une chose remarquable que la critique n'ait pas su déceler sa présence sous la féminisation de son nom, Joan Ruskin ${ }^{24}$. Le rapprochement de ces deux noms est on ne peut plus évident lors de l'enquête bizarre de la Sûreté cantonale vaudoise devant Ghezzo-Quénum:

Mais je vous assure que cet homme est dangereux, qu' en ce moment même il a peut-être réussi à attirer ma femme dans un piège et il va peut-être l'étrangler - de la même façon qu'il a étranglé Joan...

- John?

- Non, Joan. Il s'agit de la sæur de Rachel. (p. 162)

John Ruskin, l'un des plus grands représentants de l'esthétique victorienne en Angleterre, abhorrait la gesticulation, le mouvement exagéré et la fioriture du baroque, car les formes ne seraient pas, selon lui, fidèles à la nature. Elles seraient par contre artificielles et par conséquent fausses. Pour Ruskin, le seul art véritable et valable est celui qui se fonde sur la vérité naturelle rendant à l'artiste les qualités nécessaires à son édification morale et à celle du public ${ }^{25}$. C'est cette esthétique périmée, qui comporte avec elle une vision sclérosée de la vérité, qui est littéralement tuée dans le texte, mise à mort symbolisée par le meurtre de Joan.

23 Remarquons qu'il s'agit ici de la première correction faite par $R R$, de sa première apparition dans le texte, donc de sa première victoire sur le texte de l'éditeur.

24 C'est S. Söderlind, loc. cit., qui essaie pour la première fois (en 1984!) de travailler le rapport de l'œuvre de John Ruskin au roman d'Aquin.

25 Cf. Joseph Milsand, l'Esthétique anglaise. Étude sur John Ruskin, Lausanne, Norwood, 1976. 
Les deux exemples de réseaux transphrasiques brièvement esquissés ici fonctionnent au niveau du macrotexte à travers des passages souvent éloignés et travaillent à démolir le sectionnement de la vérité stratifiée. Dans ce contexte, il y a lieu de présenter également quelques exemples qui fonctionnent au niveau du microtexte en tant que calembour. Ceux-ci ont déjà été en partie étudiés par F. Iqbal ${ }^{26}$. Nous fournirons ici d'autres exemples que cette dernière n'a pas traités:

Elle a préparé une solution d'extrait de liquide anté-cervical du rat de Suède et extrait d'atropine animale (effet: sédation lente des centres nerveux, affaissement des centres médullaires et élimination complète en moins de 24 heures). Comme une vraie pupille de la First Church, Joan s'est empressée de coller une étiquette marquée "poison» sur l'éprouvette qui contenait, en quelque sorte, mon propre liquide antécervical. (p. 67. Nous soulignons)

[...] j'ai posé ma main, en guise de véronique, sur sa bouche inspirée, induisant, sans aucun doute, ma compagne sur la voie de l'enfer anglican, car elle a expiré en état de faute inavouable à son gracious Lord. Le péché est mortel, d'ailleurs, c'est tout dire! Le lendemain matin - le lundi des esclaves! -, embrumé par une surdose de véronalide, je n'ai mème pas perçu la sonnerie de mon réveille-matin [...] (p. 70. Nous soulignons).

At the end of my moonlike revolution (pas la tienne, mon chou!), je me sens toujours très sad. (p. 91)

[...] et je sais, de science certaine, que l'âme de Joan n'erre ${ }^{27}$ pas comme une âme en peine entre Elseneur et Montréal, entre le Lagos Memorial Hospital et le Redfern Memorial (désigné, de temps immémorial, parce qu'un certain Redfern a dû crever sur le parquet de sa bourse en hurlant comme un macaque Rhésus en train d'éjaculer!), entre McGill et le 27 mai, jour des jours!, ni, entre chien et loup, ni à plus forte raison, entre ce chien de Montcalm et Wolfe! (p. 85. Nous soulignons)

Dans le premier exemple, sont réunis dans un seul passage deux aspects très importants du domaine de la pharmacie, celui de l'expérience scientifique et celui de l'effet produit sur un malade, les deux fonctionnant au niveau d'une leçon à administrer à une élève (pupille). Le calembour est un peu caché dans la mesure où l'atropine est un alcaloïde, extrait de la belladone, utilisé en médecine pour dilater la pupille (oculaire).

26 Françoise Iqbal, Hubert Aquin, romancier, Québec, Presses de l'université Laval, 1978, p. 154-159.

27 Le verbe «errer» est explicitement rattaché à RR: et maintenant je pense follement $\grave{a} R R$ qui erre, erre en ce moment, loin de moi sur les quais [...] (p. 154) Ici, Joan erre, elle aussi, et les liens secrets qui unissent les deux sœurs deviennent lentement visibles. 
Dans le deuxième exemple que nous avons relevé, «véronique», passe du matador exécutée avec sa cape, appelle le barbiturique véronal et ainsi sont réunis deux univers discursifs du roman, celui de la drogue et celui de la tauromachie (cf. plus loin p. 128).

Les troisième et quatrième exemples contiennent des calembours bilingues. Dans le troisième, il faut penser à l'expression québécoise «les lunes» qui veut dire «les menstrues» pour comprendre le sens de l'anglais. Ce calembour caché permet en'tout cas de rendre explicites les liens souvent soulignés dans le texte entre la femme et le pays.

Le quatrième exemple que nous avons reproduit ici travaille d'abord le motif fondamental de la mémoire que nous examinerons plus loin. Ce passage introduit également un glissement à partir du chien et du loup (voir aussi la chienne de Joan) vers l'histoire de la défaite québécoise. Ces exemples indiquent amplement la façon dont sont combinés différents personnages et différents univers discursifs pour créer ce que nous avons appelé des métaphores transphrastiques.

Un autre réseau de la métaphore transphrastique, présent à la fois en tant que calembour au niveau du microtexte et en tant qu'élément structurant au niveau du macrotexte, est la séquence phonétique [sê] qui se trouve dans les mots clefs suivants: cinq, saint, sain, sein, singe, assassin. La fréquence considérable de ces éléments permet la conjonction des thèmes et des personnages les plus importants du roman. Il en est de même pour le réseau constitué par les paronymes suivants: cri, crime, Christ, écris, crie, Cri.

Ces réseaux sont constamment réactivés à partir d'un seul élément qui rappelle ses cousins. Il en résulte que l'emploi d'un seul élément d'un réseau donné permet de sentir la présence simultanée et souterraine d'une multitude de sèmes et de plusieurs réseaux hétéroclites. Se créent ainsi dans le roman des effets spéciaux de distanciation dans la mesure où une lecture attentive est capable d'emmagasiner un étalage impressionnant d'éléments réactivés à des moments divers.

Reprenons maintenant la question de la mémoire que nous venons d'évoquer à propos du quatrième exemple. Trou de mémoire fonctionne au niveau de la surface pour faire place à la volonté d'imposer une vérité policière, de remplir tous les trous, toutes les ellipses, toutes les omissions. À un niveau plus profond, nous voyons la futilité de cette entreprise, son impossibilité même. Nous croyons que l'attitude à examiner face à la mémoire est celle de RR. C'est elle qui vit le plus intensément les dures leçons de son passé et qui est obligée de les revivre mille fois sous l'effet de la drogue et de l'écriture. Pierre X. Magnant et Ghezzo-Quénum essaient, par contre, de tout oublier:

Aie... Si seulement je pouvais me laver le cerveau avec un superdétergent à action mousseuse instantanée et ne plus revoir, au centre de mon insomnie, le corps blanc de Joan dont le poids mort et la présence folle m'accablent et me tuent. (p. 51) 
Ah que ma mémoire se casse enfin, que la vie s'effrite et que cet édifice incertain de souvenirs soit réduit à néant, car je n'en peux plus! (p. 190)

L'éditeur, par exemple, tente en vain de disqualifier le récit de RR qui s'ajoute au sien et lui enlève sa position de premier lecteur. Les fois où il essaic de reconstituer le passé, il le fait dans un but improductif qui ne cherche qu'à remplir les trous dont il a peur. RR, par contre, se fait raconter un passé traumatique qu'elle a oublié et elle se laisse transformer par cette remémoration. Les personnes opprimées doivent reconstituer leur enfance dans le seul but d'en finir avec leur enfance (p. 63), et ceci en accord avec la dialectique du colonisé et de son passé culturel étudiée par Frantz Fanon ${ }^{28}$ : une culture une fois retrouvée ne sera jamais identique à elle-même, elle se sera transformée du fait d'avoir été retrouvée et du fait de renaître dans un nouveau contexte. RR revit le passé et se laisse transformer dans l'écriture. Elle vit et revit sans cesse cette expérience en tant qu'expćrience vivante.

En ceci, l'écriture joue dans ce roman un rôle bien curieux. Car oublicr par l'écriture, chose entreprise par Pierre X. Magnant, par exemple, est une tâche impossible. Le fait d'essayer d'oublier quelque chose rappclle précisément la chose qu'on veut oublier. Le récit de P.X. Magnant peut donc sc comprendre comme une énorme prétérition écrite. C'est un texte qui laisse apparaître ce qu'il ne voulait pas dire. Car le geste écrit est un geste permanent qui ne s'efface que difficilement. Voilà ce qui donne sens aux déclarations finales de RR à propos de son futur enfant. N'oublions pas qu'elle laisse paraitre ce texte compliqué malgré son désir que l'enfant n'apprenne jamais son passé. Ce dernier n'aura plus besoin de se tourner vers le passé: il représente par contre l'avenir, un futur ćditcur du texte. Il figurera un nouvel éditeur dans une longue sćrie d'éditeurs et de lecteurs. Car le geste écrit de sa mère est, malgré elle, pcrmanent - mais peut-être pourra-t-il profiter de la transformation possible, comme l'a fait d'aillcurs sa mère avant lui, au licu de se scléroser dans une vision morte de son passé, à la manière de tant d'autres avant lui.

La permanence du geste écrit de RR, malgré ce désir de tout cacher à son enfant, n'est qu'un exemple parmi tant d'autres des incohérences de la prétendue version finale de RR. Pourquoi des dates de Ghezzo-Quénum ne collentelles pas? Pourquoi Ghezzo-Quénum n'aurait-il pas remarqué plus tôt l'adresse 123, rue Saint-Sacrement où il avait déjà écrit? Quand l'éditeur aurait-il lu le journal de Ghezzo-Quénum? Quand aurait-il écrit les notes sur la dernière convcrsation? De qui sont les notes non signées du texte? Avec tant de questions déroutantes, comment croire que RR est l'éditrice finale? La série d'éditcurs doit nécessairement continuer. Car les personnages de Trou de mémoire sont profondément dostoïevskiens, au sens où Bakhtine entend le personnage ${ }^{29}$. Ils

28 Frantz Fanon, les Damnés de la terre, Paris, Maspéro, 1961, passim.

29 Voir à ce propos Anthony Wall, «Characters in Bakhtin's Theory», Studies in Twentieth Century Literature, no 9, 1984, p. 41-56. 
ont toujours déjà lu le roman avant nous, les lecteurs, et ils anticipent toujours nos objections. En ceci, nous pouvons être pleinement d'accord avec J.-P. Martel qui dit que le personnage d'Aquin viole le lecteur dans son droit à la lecture première et solitaire. ${ }^{30} \mathrm{Vu}$ de l'extérieur, Trou de mémoire se conçoit comme une série interminable d'extensions du texte où chaque personnage essaie, à tour de rôle, d'avoir le dernier mot $^{31}$ :

$J$ 'ai presque tué à jamais les mots que Joan a proférés dans la phase précomateuse de son intoxication: elle n'a jamais cessé de monologuer comme un anglican pacifiste, elle n'en finissait plus de me faire part de nos projets d'amour et de proférer des dernières paroles que d'autres, plus finales encore, rendaient anté-pénultièmes et ainsi de suite, ce qui n'est pas conforme au mutisme des Anglais. (p. 86-87)

Vu pourtant de l'extérieur, Trou de mémoire constitue une expérience active de lecture dont les limites s'étendent sans cesse avec toutes les expansions et contestations des multiples récits et niveaux diégétiques. La ligne qui sépare le récit des notes infrapaginales n'est nullement une frontière infranchissable entre la fiction et l'objectivité, car en effet, ces deux espaces se recoupent constamment. Ils se parlent et ils se contestent. Les notes servent à fournir un cadre superficiel à la tentative visant à stratifier la vérité vitale, tentative qui s'avère très tôt infructueuse. Le roman conteste donc la prétention à la vérité absolue dont se réclame la note: cette vérité du policier détective qu'elle représente plutôt est un idéal qui nous empêche de vivre et nous force à observer la vie de l'extérieur. C'est finalement la vérité des singes du laboratoires qui sont incapables d'agir. Les singes, comme d'ailleurs les signes, ne sauront pas parler à ceux qui vivent, tels les policiers empiriques du texte, à l'extérieur du mouvement de la vie.

Les policiers vivent dans une telle platitude qu'il suffit d'une touche d'excentricité et d'une menace réelle (mais, à leurs yeux, anormale) pour qu'ils soient enclins à tout soupçonner, y compris le premier individu qui vient les en informer. (p. 164)

Les policiers, c'est connu, ne prennent jamais les singes au sérieux. (p. 28)

La vie bouge et la vérité vitale est précisément l'expćrience de ce mouvement. Cette vérité est sans limites, elle ne se capte ni par le compartimentage ni par la hiérarchisation: elle se vit. Comme l'écrit Patricia Smart: L'absolu

30 J.-P. Martel, loc. cit., p. 83.

31 Relisons le passage suivant de Mikhail Bakhtine, la Poétique de Dostoïevski, Paris, Seuil, 1970, p. 301-302, à propos de la problématique du dernier mot dans le roman: L'échappatoire est la possibilité qu'on se réserve de modifier le sens final, déterminant, de son mot; ce qui se reflète nécessairement dans sa structure. L'autre sens éventuel, c'est-à-dire la sortie qu'on se ménage, accompagne comme une ombre chaque mot. D'après sa signification, le mot avec échappatoire doit être le dernier mot et se fait passer effectivement pour tel, mais n'est en réalité que l'avant-dernier et place derrière soi un point final purement conventionnel. 
n'apparaît plus comme un but statique, mais plutôt comme un "point de fuite» qui s'éloigne à mesure qu'il s' en approche. 32

L'esthétique aquinienne du mouvement vécu a déjà été largement décrite en termes du baroque ${ }^{33}$. Nous cherchons ici à faire sentir le fait que le déploiement de tant de mouvement repose non seulement sur un principe esthétique, mais, plus profondément, sur une compréhension de l'expérience comme activité sans arrêt. Trou de mémoire constitue une expérience esthétique et philosophique qui sera toujours opposée à la vision de stabilité proposée par l'art victorien ainsi que le conçoit John Ruskin. Le roman peut d'abord sembler intenable dans la mesure où nous courons toujours après la vérité des mots qui ont précédé et que nous arrivons toujours inéluctablement quelques secondes en retard. Mais l'essence de l'art aquinien se trouve précisément dans le mouvement constant et dans la destruction de toute frontière fixe qui essaie de le restreindre. C'est en ceci qu'il reste un art profondément lié aux procédés sémiotiques de la métaphore qui sont sans cesse utilisés pour dire l'absolu, la vérité. Mais l'absolu ne se dit jamais, car il est innommable. Alors, il nous incite éternellement à tourner devant lui. Seulement en rond? Qui saurait le dire avec certitude?

32 Patricia Smart, Hubert Aquin, agent double, Montréal, Presses de l'Université de Montréal, 1973, p. 98.

33 J.-P. Martel, loc. cit., passim. 